\title{
Subsecond Ensemble Dynamics of Orexin Neurons Link Sensation and Action
}

\author{
Denis Burdakov
}

Department of Health Sciences and Technology, ETH Zürich, Zürich, Switzerland

\begin{abstract}
Hypothalamic hypocretin/orexin neurons have been initially conceptualized as slow, modulatory controllers of behavior. Furthermore, their behavioral effects have been assumed to be a secondary consequence of their impact on arousal. However, cellular-resolution calcium imaging and optogenetic studies show that orexin neurons regulate self-generated and sensory-evoked movement on rapid, subsecond timescales. Orexin cell activity rapidly and transiently peaks before and during movements. Optogenetic prevention of this activation reduces the probability of locomotion initiation, and optogenetic mimicry of orexin cell activation rapidly causes locomotion. Neural ensemble calcium imaging experiments reveal that the same orexin cells whose activity underlies movement initiation display subsecond-latency responses to diverse sensory stimuli. These findings establish orexin neurons as rapid and strong sensorimotor controllers that are in many ways operationally similar to classic subcortical movement controllers, such as midbrain dopamine neurons. While a scientific definition of "arousal" is still lacking, the subsecond-scale sensorimotor control by orexin neurons could be viewed as reminiscent of a motor rather than an arousal system.

(C) 2021 The Author(s)

Published by S. Karger AG, Basel
\end{abstract}

Brain function relies on context-appropriate changes in the dynamics of neuronal electrical membrane potential (action potential firing). This is the primary determinant of neurotransmitter release, including hypocretin/orexin transmitters. The firing of hypocretin/ orexin-released neurons (orexin neurons), and its effects on postsynaptic targets, is therefore of central interest for understanding the role of orexin neurons in brain function and has been intensely researched. These studies revealed that orexin cell firing is modulated 
by multiple neurotransmitters and also produces and releases (together with orexin peptides) several neurotransmitters such as dynorphin and glutamate [1-30]. Since the end of 2010, it has become possible to simultaneously visualize real-time activity dynamics of multiple orexin neurons (ensemble dynamics) in the brains of behaving animals, revealing new levels of cellular heterogeneity in the orexin system and its relation to rapidlychanging behavior [16]. The latter phenomena form the focus of this chapter, after a brief introduction.

In vitro electrophysiological recordings from rodent orexin neurons, identified either by postrecording immunolabelling or transgenic insertion of green fluorescent protein, have consistently revealed that orexin neurons are intrinsic pacemakers that generate tonic firing at around $10 \mathrm{~Hz}$, in a manner resistant to blockers of fast synaptic inputs [1, $7,8,13,25,28,31-35]$. This tonic firing is slowly (minutes) modulated by nutrients, gasses, neuromodulators, and possibly local electrical oscillations $[1,11,14,15,17,18,25,26$, 36-38]. In vivo, the firing dynamics of orexin neurons can be very different. As detailed in this chapter, they change rapidly (milliseconds) in response to sensory stimuli and/or motor actions. As described below, these rapid changes appear important for normal sensorimotor control at the subsecond timescale. In contrast, the relative contribution to in vivo orexin cell activity of the slow modulators (nutrients, gasses) remains to be defined, and will not be discussed further here. This is, however, an important subject for future research.

One key function of the brain is to create movements that enable foraging and appropriately connect sensations to actions. Here, the role of the cortex for rapid (subsecond) sensorimotor transformations is well documented [39]. On the other hand, regions such as the hypothalamus are traditionally not conceptualized as direct players in rapid sensorimotor control, except from the perspective of gating perception via (slowly changing) arousal.

Yet, since 2005, orexin neurons have repeatedly been reported to change their in vivo firing dynamics on a subsecond timescale, including in response to visual, somatosensory, and olfactory stimulation, as well as in the context of rapid movements. Specifically, electrophysiological action potential recordings, and averaged neural population recordings with low-resolution optical methods, have revealed that orexin neurons' activity represents external sensory stimuli within milliseconds and that orexin cell activity correlates with muscle/electromyography activation [3, 12, 29, 40-43]. At least some of the rapid sensory responses occur in orexin neurons; however, none occur in neighboring lateral hypothalamic neurons expressing the melanin-concentrating hormone [12]. This suggests that orexin cells are specialized for receiving external sensory information $[12,19]$. These findings contrast with the earlier conceptualization of orexin neurons as sensors of only slowly changing levels of hormones and nutrients, and chronic/slow controllers of arousal and movement [26, 44-46].

If the main function of orexin neurons is slow control of arousal, then it is not clear why they need to change their firing on a subsecond timescale in order to implement this function. If orexin neurons only perform slow/modulatory control of arousal and move- 
ment, what then is the purpose of the observed subsecond changes in their activity in vivo? The activity of orexin neuron ensembles in vivo was not recorded in the above studies. Therefore, it remained unclear whether the majority of orexin neurons change their activity in association with fast sensations and movements. The physiological significance, in particular the causal role in actions/movements, of sensory responses of orexin neurons was also not elucidated. These knowledge gaps were addressed by more recent experiments [16], which assessed the behavioral correlates of orexin neuron ensemble activity and examined how rapidly orexin neurons can control awake behavior in response to sensory input.

Neuronal ensemble activity of orexin neurons can be visualized at cellular resolution and in real-time using three-dimensional ("volumetric") 2-photon laser scanning calcium imaging [16]. Such imaging of $>300$ orexin neurons in mice, performed simultaneously with precise quantification of locomotion using a treadmill, suggested that activation of the majority $(\sim 70 \%)$ of orexin neurons correlated with initiation of locomotor bouts (Fig. 1a-c). A closer inspection of the temporal correspondence of the orexin cell activity vector and the locomotion speed revealed that the majority of orexin neurons increase their activity up to several seconds prior to locomotion initiation [16]. In fact, based on the activity vector of orexin cells, a simple machine learning approach was able to accurately predict imminent locomotion initiation (Fig. 1d).

The temporal order of neurobehavioral dynamics (orexin cell activity first, running second) in these experiments is consistent with the idea that activation of at least some orexin neurons may rapidly cause running. This would imply that orexin neurons control behavior, not only through a slow (10s of seconds) modulation of "arousal" - placed here in parentheses due to lack of a scientific definition of such a term (commented on further below) - but also via rapid direct activation of motor systems, as expected from electrophysiological data [47] on orexin circuits.

Direct evidence for this rapid coupling between orexin cell firing and behavior comes from optogenetic stimulation experiments that examined whether stimulation of action potential firing in orexin neurons can elicit movement. Optogenetics involves the use of light to stimulate or inhibit cells that have been genetically modified to express light-sensitive proteins, and/or to measure the response of these cells to stimulation [48]. Indeed, optogenetic excitation of orexin cell firing above around $7 \mathrm{~Hz}$, a frequency consistent with their natural firing rates in vivo $[3,29]$, led to frequency-dependent stimulation of running. In these experiments, the latencies between the increase in orexin cell firing and locomotion onset were in the range of 300-4,880 ms, with a median of $1.75 \mathrm{~s}$ [16], indicating that orexin cell activation leads to rapid initiation of movement (Fig. 1e).

Conversely, optogenetic silencing of orexin neurons reduces the probability of locomotion initiation [16]. The ability of orexin cell firing to rapidly initiate locomotion, together with the fact that orexin cell firing is rapidly ( $<100 \mathrm{~ms})$ recruited by sensory stimuli of multiple modalities [16], raises the question of whether orexin cells are causally involved in fast sensorimotor transformations. This has been examined by optogenetic silencing experiments where reversible orexin cell inactivation was temporally targeted to 


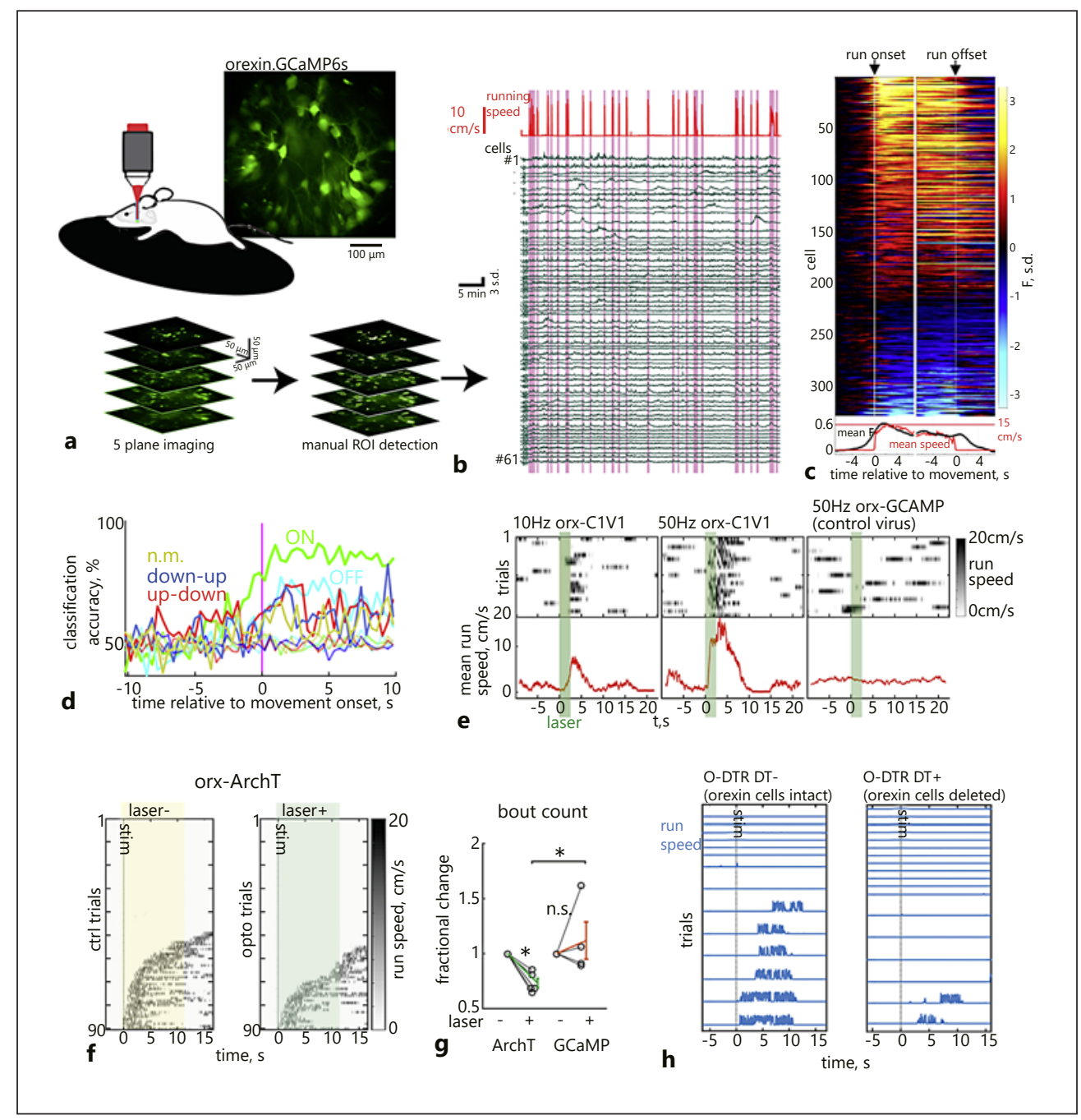

Fig. 1. Locomotor correlates of orexin neural ensemble dynamics. a Experimental set-up. b Examples of orexin cell ensemble dynamics in relation to running. c Orexin cell activity aligned to start and end of running bouts. $\mathbf{d}$ Machine learning accuracy to predict locomotion bouts based on activity of different orexin cell types. e Effect of optogenetic stimulation of orexin cells on running. $\mathbf{f}$ Effect of optogenetic inhibition of orexin cells on running. $\mathbf{g}$ Running bout quantification from the experiment in $\mathbf{f}$. h Effect of orexin cell deletion (O-DTR D+) on sensory-evoked running. All data are from [16], where further details are given. F, s.d, calcium fluorescence Z-score, s.d.; GCaMP6s, genetically encoded $\mathrm{Ca}^{2+}$ indicator; ROI, region of interest; n.m., not modulated. Reprinted with permission under the Creative Commons Attribution 4.0 International (CC BY 4.0) License (https://creativecommons.org/licenses/ by/4.0/) from Karnani et al. [16].

the precise moments of sensation. Examination of sensory-evoked locomotion initiation in these experiments reveals that mice are significantly less likely to initiate locomotion when the sensory-evoked activity of orexin cells is disrupted (Fig. 1f, g). Therefore, rapid dynamics of orexin neurons is critical for mediating rapid sensorimotor transformations. 
Mice with deleted orexin neurons also display an inability to generate normal running responses upon sensory stimulation (Fig. 1h) [16].

These data provide causal evidence for a role of orexin neurons in subsecond-scale sensorimotor transformations and self-paced movements [16]. This contrasts with much of the previous literature on orexin cells, which has examined primarily the slower modulation by orexin neurons of behavioral states such as arousal and obesity. While previous work has also found that orexin neurons display rapid sensory responses [3, 12, 40, 41], the motor importance of these subsecond activity fluctuations had been unclear until the study of Karnani et al. [16]. Interestingly, several further studies have begun to demonstrate that rapid dynamics of other lateral hypothalamic neurons are also causally linked to rapid changes in behavior [49-52].

Although arousal is a vague term that lacks a clear scientific definition and has been used to refer to diverse brain and motor states (e.g., wakefulness, movement, attention, goal-oriented actions, sex), studies had previously implied that orexin neurons contribute to the arousal process via a slow, modulatory influence [44]. In contrast, Karnani et al. [16] found that orexin cell firing evokes locomotion in milliseconds. This finding suggests that orexin cells in fact play a rapid role in locomotion initiation, similar to established motor control centers such as the midbrain dopamine cells [53]. The demonstration that subsecond sensory responses of orexin cells are causally involved in rapid control of locomotor output, similar to sensorimotor transformations in the neocortex [54, 55], clarifies the need to update orexin neurons sensory representations on a subsecond timescale. Do orexin neurons control specific phases of locomotion? Temporally targeted acute optogenetic silencing experiments suggest a specific role in locomotion initiation, rather than locomotion maintenance [16].

In order to convert these new findings into an integrated scientific concept for the role of the orexin system in brain function, a precise scientific definition of "arousal" may be required. For any neuron that effects movement, the labeling of its function as "arousal" depends on the temporal definition of arousal, which is missing from most of the current literature. The timescale of orexin neuron activation by external stimuli can be as short as $34 \mathrm{~ms}$, and orexin neuron activation can produce movements as rapidly as $300 \mathrm{~ms}$ [16]. Irrespective of whether these effects are called "arousal" or "sensorimotor control," it is clear that orexin neurons, in addition to their paradigmatic position as slow regulators of body physiology, exert rapid control over motor performance. It would be interesting to investigate whether orexin neurons provide parallel inputs to arousal and motor systems, which may ensure that attention and movement occur together.

Many questions remain unanswered regarding the rapid motor effects of orexin neuron activation. For example, which neurotransmitters and postsynaptic targets mediate these effects? How do they relate to the existing view of the hypocretin/orexin system as a slow arousal controller $[5,6,20,21,23,24,37,56-63]$ ? It is tempting to speculate that, given the rapid (millisecond) speed of glutamate action on its ionotropic receptors compared with the slow (sec-min) G-protein-coupled receptor signaling of orexin peptides, the rapid motor actions of orexin cells are mediated by glutamate release from orexin cell 
axons $[21,22,64]$. However, the role of orexin peptides in these effects cannot yet be excluded. This is because lateral hypothalamic activation can generate orexin-receptor-dependent engagement of spinal motoneurons with $<100 \mathrm{~ms}$ delay [47].

Understanding the relative physiological roles of co-released orexin and non-orexin neurotransmitters has important implications for drug design. For example, drugs targeting orexin receptors, such as those being developed for insomnia [65, 66], may preserve the functions of orexin neurons that are mediated by glutamate. Human narcoleptic patients, who are thought to lack orexin neurons (and hence, not only orexin transmitters but all other transmitters synthesized in orexin neurons), display severe abnormalities in context-appropriate motor control such as dramatic postural collapse in response to certain sensory stimuli [57]. It is currently unclear whether this human pathology is entirely due to orexin deficiency, or whether the loss of other transmitters made by orexin cells also contributes.

In summary, recent data suggest that orexin neurons can be added to a growing network of known movement-controlling neurons in the brain [55]. It is currently unclear whether this means that movement-control involves neural systems with a certain redundancy between them, or whether hitherto-used experimental readouts of movement are simply inadequate for distinguishing between non-redundant neural controllers. So far, there appear to be striking similarities between the roles in movement of orexin and dopamine systems. For example, da Silva et al. [53] found that midbrain dopamine neurons are causally involved in locomotion initiation and have diverse activity profiles during movement, with some cells turning off and some on, similar to what is observed in orexin neurons [16]. Also similar to orexin neurons, the established movement-control neurons in the striatum activate before self-initiated actions [67]. Given these similarities, would it be more accurate to refer to the orexin system as a "motor" rather than an "arousal" system? Interestingly, the reverse question has been raised for midbrain dopamine circuits, which were found to have the sleep-wake effects expected of an arousal system [68]. Further conceptual progress in this field may require a modernization of our scientific terminology used to describe the different timescales at which neural dynamics are coupled to brain state and behavior.

\section{Key Take-Home Points}

- Orexin cell activity is necessary for initiation of normal locomotor responses to external sensory stimuli.

- The activity of most (but not all) orexin cells changes rapidly before and during movements.

- Most orexin neurons rapidly (subsecond) respond to external sensory stimuli.

\section{Conflict of Interest Statement}

D.B. received consultancy fees from Idorsia Pharmaceuticals Ltd during the development of this book. He has no other conflict of interest to declare. 


\section{Funding Sources}

The work in the author's laboratory has been funded by SNSF, ETH Zürich, ERC-FP7, and HFSP.

\section{References}

1 Li Y, Gao XB, Sakurai T, van den Pol AN: Hypocretin/ Orexin excites hypocretin neurons via a local glutamate neuron-A potential mechanism for orchestrating the hypothalamic arousal system. Neuron 2002;36:11691181.

2 Burdakov D: Electrical signaling in central orexin/hypocretin circuits: tuning arousal and appetite to fit the environment. Neuroscientist 2004;10:286-291.

3 Mileykovskiy BY, Kiyashchenko LI, Siegel JM: Behavioral correlates of activity in identified hypocretin/orexin neurons. Neuron 2005;46:787-798.

4 Hassani OK, Lee MG, Jones BE: Melanin-concentrating hormone neurons discharge in a reciprocal manner to orexin neurons across the sleep-wake cycle. Proc Natl Acad Sci USA 2009;106:2418-2422.

5 Blomeley C, Garau C, Burdakov D: Accumbal D2 cells orchestrate innate risk-avoidance according to orexin signals. Nat Neurosci 2018;21:29-32.

6 Apergis-Schoute J, Iordanidou P, Faure C, Jego S, Schone C, Aitta-Aho T, et al: Optogenetic evidence for inhibitory signaling from orexin to $\mathrm{MCH}$ neurons via local microcircuits. J Neurosci 2015;35:5435-5441.

7 Burdakov D, Alexopoulos H, Vincent A, Ashcroft FM: Low-voltage-activated A-current controls the firing dynamics of mouse hypothalamic orexin neurons. Eur J Neurosci 2004;20:3281-3285.

8 Burdakov D, Gerasimenko O, Verkhratsky A: Physiological changes in glucose differentially modulate the excitability of hypothalamic melanin-concentrating hormone and orexin neurons in situ. J Neurosci 2005;25: 2429-2433.

9 Burdakov D, Jensen LT, Alexopoulos H, Williams RH, Fearon IM, O'Kelly I, et al: Tandem-pore K+ channels mediate inhibition of orexin neurons by glucose. Neuron 2006;50:711-722.

10 Burdakov D, Karnani MM: Ultra-sparse connectivity within the lateral hypothalamus. Curr Biol 2020;30: 4063-70.e2.

11 Gonzalez JA, Horjales-Araujo E, Fugger L, Broberger C, Burdakov D: Stimulation of orexin/hypocretin neurones by thyrotropin-releasing hormone. J Physiol 2009; 587(Pt 6):1179-1186.

12 Gonzalez JA, Iordanidou P, Strom M, Adamantidis A, Burdakov D: Awake dynamics and brain-wide direct inputs of hypothalamic MCH and orexin networks. Nat Commun 2016;7:11395.

13 Gonzalez JA, Jensen LT, Doyle SE, Miranda-Anaya M, Menaker M, Fugger L, et al: Deletion of TASK1 and TASK3 channels disrupts intrinsic excitability but does not abolish glucose or $\mathrm{pH}$ responses of orexin/hypocretin neurons. Eur J Neurosci 2009;30:57-64.
14 Gonzalez JA, Jensen LT, Fugger L, Burdakov D: Metabolism-independent sugar sensing in central orexin neurons. Diabetes 2008;57:2569-2576.

15 Karnani MM, Apergis-Schoute J, Adamantidis A, Jensen LT, de Lecea L, Fugger L, et al: Activation of central orexin/hypocretin neurons by dietary amino acids. Neuron 2011;72:616-629.

16 Karnani MM, Schone C, Bracey EF, Gonzalez JA, Viskaitis P, Li HT, et al: Role of spontaneous and sensory orexin network dynamics in rapid locomotion initiation. Prog Neurobiol 2020;187:101771.

17 Karnani MM, Venner A, Jensen LT, Fugger L, Burdakov D: Direct and indirect control of orexin/hypocretin neurons by glycine receptors. J Physiol 2011;589(Pt 3):639651.

18 Kosse C, Burdakov D: Fast and slow oscillations recruit molecularly-distinct subnetworks of lateral hypothalamic neurons in situ. eNeuro 2018;5:ENEURO.0012-18.2018.

19 Kosse C, Burdakov D: Natural hypothalamic circuit dynamics underlying object memorization. Nat Commun 2019;10:2505.

20 Kosse C, Schone C, Bracey E, Burdakov D: Orexin-driven GAD65 network of the lateral hypothalamus sets physical activity in mice. Proc Natl Acad Sci USA 2017; 114:4525-4530.

21 Schöne C, Apergis-Schoute J, Sakurai T, Adamantidis A, Burdakov D: Coreleased orexin and glutamate evoke nonredundant spike outputs and computations in histamine neurons. Cell Rep 2014;7:697-704.

22 Schöne C, Burdakov D: Glutamate and GABA as rapid effectors of hypothalamic "peptidergic" neurons. Front Behav Neurosci 2012;6:81.

23 Venner A, Karnani MM, Gonzalez JA, Jensen LT, Fugger L, Burdakov D: Orexin neurons as conditional glucosensors: paradoxical regulation of sugar sensing by intracellular fuels. J Physiol 2011;589(Pt 23):5701-5708.

24 Williams RH, Alexopoulos H, Jensen LT, Fugger L, Burdakov D: Adaptive sugar sensors in hypothalamic feeding circuits. Proc Natl Acad Sci USA 2008;105:1197511980.

25 Williams RH, Jensen LT, Verkhratsky A, Fugger L, Burdakov D: Control of hypothalamic orexin neurons by acid and CO2. Proc Natl Acad Sci USA 2007;104:1068510690.

26 Yamanaka A, Beuckmann CT, Willie JT, Hara J, Tsujino $\mathrm{N}$, Mieda M, et al: Hypothalamic orexin neurons regulate arousal according to energy balance in mice. Neuron 2003;38:701-713.

27 Grivel J, Cvetkovic V, Bayer L, Machard D, Tobler I, Muhlethaler M, et al: The wake-promoting hypocretin/ orexin neurons change their response to noradrenaline after sleep deprivation. J Neurosci 2005;25:4127-4130. 
28 Eggermann E, Bayer L, Serafin M, Saint-Mleux B, Bernheim L, Machard D, et al: The wake-promoting hypocretin-orexin neurons are in an intrinsic state of membrane depolarization. J Neurosci 2003;23:1557-1562.

29 Lee MG, Hassani OK, Jones BE: Discharge of identified orexin/hypocretin neurons across the sleep-waking cycle. J Neurosci 2005;25:6716-6720.

30 Aitta-Aho T, Pappa E, Burdakov D, Apergis-Schoute J: Cellular activation of hypothalamic hypocretin/orexin neurons facilitates short-term spatial memory in mice. Neurobiol Learn Mem 2016;136:183-188.

31 Yamanaka A, Muraki Y, Tsujino N, Goto K, Sakurai T: Regulation of orexin neurons by the monoaminergic and cholinergic systems. Biochem Biophys Res Commun 2003;303:120-129.

32 van den Pol AN, Gao XB, Obrietan K, Kilduff TS, Belousov AB: Presynaptic and postsynaptic actions and modulation of neuroendocrine neurons by a new hypothalamic peptide, hypocretin/orexin. J Neurosci 1998; 18:7962-7971.

33 Schöne C, Venner A, Knowles D, Karnani MM, Burdakov D: Dichotomous cellular properties of mouse orexin/hypocretin neurons. J Physiol 2011;589(Pt 11):27672779.

34 Williams RH, Morton AJ, Burdakov D: Paradoxical function of orexin/hypocretin circuits in a mouse model of Huntington's disease. Neurobiol Dis 2011;42:438455.

35 Williams RH, Burdakov D: Silencing of ventromedial hypothalamic neurons by glucose-stimulated $\mathrm{K}(+)$ currents. Pflugers Arch 2009;458:777-783.

36 Gonzalez JA, Horjales-Araujo E, Fugger L, Broberger C, Burdakov D: Stimulation of orexin/hypocretin neurones by thyrotropin-releasing hormone. J Physiol 2009; 587(Pt 6):1179-1186.

37 Gonzalez JA, Reimann F, Burdakov D: Dissociation between sensing and metabolism of glucose in sugar sensing neurones. J Physiol 2009;587(Pt 1):41-48.

38 Carus-Cadavieco M, Gorbati M, Ye L, Bender F, van der Veldt S, Kosse C, et al: Gamma oscillations organize top-down signalling to hypothalamus and enable food seeking. Nature 2017;542:232-236.

39 Crochet S, Lee SH, Petersen CCH: Neural circuits for goal-directed sensorimotor transformations. Trends Neurosci 2019;42:66-77.

40 Gonzalez JA, Jensen LT, Iordanidou P, Strom M, Fugger L, Burdakov D: Inhibitory interplay between orexin neurons and eating. Curr Biol 2016;26:2486-2491.

41 Hassani OK, Krause MR, Mainville L, Cordova CA, Jones BE: Orexin neurons respond differentially to auditory cues associated with appetitive versus aversive outcomes. J Neurosci 2016;36:1747-1757.

42 Takahashi K, Lin JS, Sakai K: Neuronal activity of orexin and non-orexin waking-active neurons during wakesleep states in the mouse. Neuroscience 2008;153:860870.

43 Burdakov D, Peleg-Raibstein D: Hypothalamic heuristics for survival. Trends Endocrinol Metab 2019;30:689691.
44 Adamantidis AR, Zhang F, Aravanis AM, Deisseroth K, de Lecea L: Neural substrates of awakening probed with optogenetic control of hypocretin neurons. Nature 2007; 450:420-424.

45 Sakurai T, Amemiya A, Ishii M, Matsuzaki I, Chemelli $\mathrm{RM}$, Tanaka H, et al: Orexins and orexin receptors: a family of hypothalamic neuropeptides and G proteincoupled receptors that regulate feeding behavior. Cell 1998;92:573-585.

46 Stanojlovic M, Pallais JP, Lee MK, Kotz CM: Pharmacological and chemogenetic orexin/hypocretin intervention ameliorates Hipp-dependent memory impairment in the A53T mice model of Parkinson's disease. Mol Brain 2019;12:87.

47 Yamuy J, Fung SJ, Xi M, Chase MH: Hypocretinergic control of spinal cord motoneurons. J Neurosci 2004;24: 5336-5345.

48 Hunter P: Shining a light on optogenetics: advances in electronics and protein engineering advance the field of optogenetics to study the activity of neuronal networks. EMBO Rep 2016;17:634-637.

49 Bracey EF, Burdakov D: Fast sensory representations in the lateral hypothalamus and their roles in brain function. Physiol Behav 2020;222:112952.

50 Herrera CG, Cadavieco MC, Jego S, Ponomarenko A, Korotkova T, Adamantidis A: Hypothalamic feed-forward inhibition of thalamocortical network controls arousal and consciousness. Nat Neurosci 2016;19:290298.

51 Jennings JH, Ung RL, Resendez SL, Stamatakis AM, Taylor JG, Huang J, et al: Visualizing hypothalamic network dynamics for appetitive and consummatory behaviors. Cell 2015;160:516-527.

52 Nieh EH, Vander Weele CM, Matthews GA, Presbrey $\mathrm{KN}$, Wichmann R, Leppla CA, et al: Inhibitory input from the lateral hypothalamus to the ventral tegmental area disinhibits dopamine neurons and promotes behavioral activation. Neuron 2016;90:1286-1298.

53 da Silva JA, Tecuapetla F, Paixao V, Costa RM: Dopamine neuron activity before action initiation gates and invigorates future movements. Nature 2018;554:244248.

54 Ferezou I, Haiss F, Gentet LJ, Aronoff R, Weber B, Petersen CC: Spatiotemporal dynamics of cortical sensorimotor integration in behaving mice. Neuron 2007;56: 907-923.

55 Svoboda K, Li N: Neural mechanisms of movement planning: motor cortex and beyond. Curr Opin Neurobiol 2018;49:33-41.

56 Burdakov D, Alexopoulos H: Metabolic state signalling through central hypocretin/orexin neurons. J Cell Mol Med 2005;9:795-803.

57 Bassetti CLA, Adamantidis A, Burdakov D, Han F, Gay S, Kallweit U, et al: Narcolepsy - clinical spectrum, aetiopathophysiology, diagnosis and treatment. Nat Rev Neurol 2019;15:519-539.

58 Burdakov D: K+ channels stimulated by glucose: a new energy-sensing pathway. Pflugers Arch 2007;454:19-27.

59 Burdakov D: How orexin signals bias action: hypothalamic and accumbal circuits. Brain Res 2020;1731: 145943. 
60 Burdakov D: Reactive and predictive homeostasis: roles of orexin/hypocretin neurons. Neuropharmacology 2019;154:61-67.

61 Kosse C, Burdakov D: A unifying computational framework for stability and flexibility of arousal. Front Syst Neurosci 2014;8:192.

62 Kosse C, Gonzalez A, Burdakov D: Predictive models of glucose control: roles for glucose-sensing neurones. Acta Physiol (Oxf) 2015;231:7-18.

63 Belle MD, Hughes AT, Bechtold DA, Cunningham P, Pierucci M, Burdakov D, et al: Acute suppressive and long-term phase modulation actions of orexin on the mammalian circadian clock. J Neurosci 2014;34:36073621.

64 Schöne C, Cao ZF, Apergis-Schoute J, Adamantidis A, Sakurai T, Burdakov D: Optogenetic probing of fast glutamatergic transmission from hypocretin/orexin to histamine neurons in situ. J Neurosci 2012;32:12437-

12443.
65 Brisbare-Roch C, Dingemanse J, Koberstein R, Hoever $\mathrm{P}$, Aissaoui H, Flores S, et al: Promotion of sleep by targeting the orexin system in rats, dogs and humans. Nat Med 2007;13:150-155.

66 Dauvilliers Y, Zammit G, Fietze I, Mayleben D, Seboek Kinter D, Pain S, et al: Daridorexant, a new dual orexin receptor antagonist to treat insomnia disorder. Ann Neurol 2020;87:347-356.

67 Cui G, Jun SB, Jin X, Pham MD, Vogel SS, Lovinger DM, et al: Concurrent activation of striatal direct and indirect pathways during action initiation. Nature 2013;494: 238-242.

68 Eban-Rothschild A, Rothschild G, Giardino WJ, Jones JR, de Lecea L: VTA dopaminergic neurons regulate ethologically relevant sleep-wake behaviors. Nat Neurosci 2016;19:1356-1366.
Denis Burdakov

Department of Health Sciences and Technology, ETH Zürich Schorenstrasse 16

8603 Schwerzenbach (Switzerland)

denis.burdakov@hest.ethz.ch 